\title{
Capillaroscopy in Psoriatic and Rheumatoid Arthritis: A Useful Tool for Differential Diagnosis
}

\author{
Dario Graceffa, ${ }^{1}$ Beatrice Amorosi, ${ }^{1}$ Elisa Maiani, ${ }^{1}$ Claudio Bonifati, ${ }^{1}$ \\ Maria Sole Chimenti, ${ }^{2}$ Roberto Perricone, ${ }^{2}$ and Aldo Di Carlo ${ }^{3}$ \\ ${ }^{1}$ Centre for the Study and Treatment of Psoriasis, Department of Clinical Dermatology, \\ San Gallicano Dermatological Institute (IRCCS), Rome, Italy \\ ${ }^{2}$ Rheumatology, Allergology and Clinical Immunology, Department of Internal Medicine, University of Rome Tor Vergata, Rome, Italy \\ ${ }^{3}$ San Gallicano Dermatological Institute, Rome, Italy
}

Correspondence should be addressed to Dario Graceffa; dag2003@libero.it

Received 27 June 2013; Accepted 17 November 2013

Academic Editor: Burkhard Leeb

Copyright (C) 2013 Dario Graceffa et al. This is an open access article distributed under the Creative Commons Attribution License, which permits unrestricted use, distribution, and reproduction in any medium, provided the original work is properly cited.

\begin{abstract}
Impairment of capillaries permeability and changes of microcirculation are associated with inflammatory arthritis. In order to demonstrate microvascular differences between psoriatic arthritis (PsA) and rheumatoid arthritis (RA) we analyzed capillaroscopic abnormalities such as megacapillaries, haemorrhages, ramifications, and avascular areas in patients affected by these two rheumatic disorders. Moreover to identify specific capillaroscopy patterns we analyzed the following parameters: venous limb diameter, arterial limb diameter, capillary loop diameter, amplitude of the capillary loop, linear density of capillaries (on $2 \mathrm{~mm}$ ), and number of twisted capillaries (on $4 \mathrm{~mm}$ ). Through a comparative morphometric analysis of capillaroscopy, our study demonstrated the presence of specific microvascular differences between PsA and RA providing an additional diagnostic tool for the differential diagnosis. We also suggest that capillaries structural abnormalities might reflect endothelial injury due to systemic inflammation during chronic arthritis.
\end{abstract}

\section{Introduction}

Psoriatic arthritis (PsA) is a chronic inflammatory disease affecting $0.04 \%-0.1 \%$ of the general population and occurs in one-third of patients with psoriasis. PsA can lead to severe bone erosions and joints destruction resulting in physical disability [1].

Nowadays, no specific laboratory tests are available for PsA; it is not always possible, therefore, confirm the diagnosis and provide an objective prognosis, especially at an early stage of disease.

The differential diagnosis between PsA and RA is very difficult in PsA patients with slight cutaneous signs and rheumatoid-like joint involvement [2-4]. Presence of citrullinated peptide antibodies has a high specificity for RA, but, among that, they are rarely seen in PsA patients [5].

Morphological and rheological changes in microcirculation have been widely demonstrated in PsA and RA patients.
Impairment of capillaries permeability and alterations in the connections between endothelium and extracellular matrix characterize these damages, resulting in abnormal structure and shape of the capillaries in the dermal papillae [6-8].

Capillaroscopy allows the study "in vivo" of morphological and functional characteristics of microcirculation. Several authors have tried to identify specific microvascular characteristics of cutaneous psoriasis and PsA; these studies were mainly conducted on the skin plaques, on synovial membrane and at nailfold level. However, the results are not univocal [9-12].

Aim of our study was to demonstrate specific microvascular differences between PsA and RA, evaluable by nailfold capillaroscopy.

We considered capillaroscopy of the psoriatic plaque not suitable for our purpose for the following reason. 
TABLE 1: Inclusion and exclusion criteria for participation in the study.

\author{
Inclusion criteria: \\ (i) Psoriasis vulgaris moderate-severe (Psoriasis Area Severity \\ Index) PASI $>6$ \\ (ii) Psoriatic arthritis diagnosed according to Classification \\ Criteria for Psoriatic Arthritis (CASPAR) \\ (iii) Rheumatoid arthritis diagnosed according to American \\ College of Rheumatology criteria (ACR) \\ Exclusion criteria: \\ (i) Nail psoriasis \\ (ii) Primary or secondary Raynaud's phenomenon \\ (iii) Acrocyanosis \\ (iv) Cardiovascular risk factors and/or coagulopathy \\ (v) Vasoactive therapies and/or anticoagulants
}

Microscopic observation of psoriatic plaque allows a frontal view of capillaries; it is therefore very difficult to establish if is displayed a single capillary or a branch of another. This artefact is particularly relevant in course of PsA where capillaries are typically branched and tortuous. It may cause a loss of accuracy in estimating capillaries density; conversely nailfold capillaroscopy providing a longitudinal view of capillary allows to follow it for the entire length.

\section{Patients and Methods}

From January 2012 to March 2013, 30 patients affected by PsA, 30 patients affected by RA, 30 patients affected by Psoriasis (Pso) without signs of arthropathy, and 30 healthy subjects as control, attending the Rheumatology Department of the University of Rome "Tor Vergata" and the Dermatology Department of San Gallicano Dermatologica Institute, were enrolled.

Diagnosis of PsA and RA was made, respectively, according to the clASsification Criteria for Psoriatic Arthritis (CASPAR) and the American College of Rheumatology criteria (ACR) $[13,14]$. Informed consent was obtained before capillaroscopy evaluation.

Inclusion and exclusion criteria used are summarized in Table 1.

Patients and healthy subjects were comparable for age, sex, main cardiovascular risk factors and other vascular diseases $(P$-values $=$ N.S. $)$. Patients treatments performed were equivalent in all groups. Patients demographic and clinical characteristics are summarized in Table 2.

Nailfold capillaroscopy was performed using a videomicroscope "Alpha Strumenti Srl, Melzo Italy" with optic probes equipped with 100x and 200x magnification contact lenses.

2.1. Morphological Study. We analyzed the principal vascular abnormalities (megacapillaries, haemorrhages, ramifications, and avascular areas) using a semiquantitative rating scale to score these changes, according to previous studies [11].

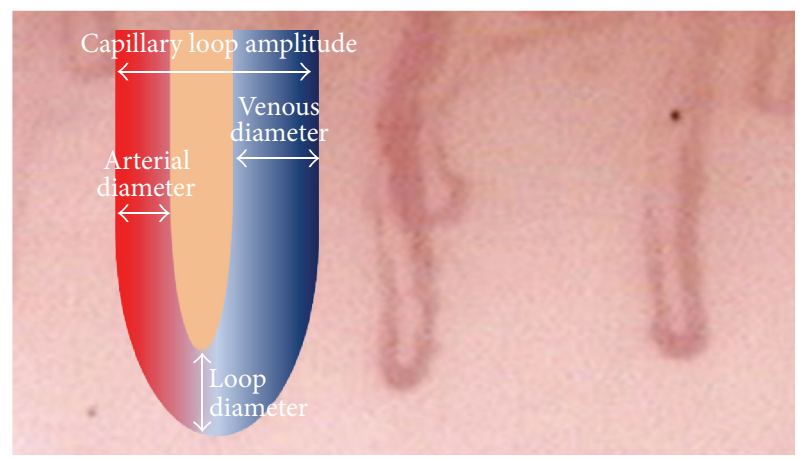

FIGURE 1: Capillary dimensions measured in the morphometric study.

2.2. Morphometric Study (Figure 1). We measured the following parameters: venous limb diameter $(\mu \mathrm{m})$, arterial limb diameter $(\mu \mathrm{m})$, capillary loop diameter $(\mu \mathrm{m})$, amplitude of the capillary loop $(\mu \mathrm{m})$, linear density of capillaries expressed as number of loops on $2 \mathrm{~mm}$, and number of twisted capillaries (on $4 \mathrm{~mm}$ ).

It was not possible to perform reliable assessments of the capillary length due to high tortuosity and difficulties in determining the angle between capillary and skin surface, especially in psoriatic patients.

Data were statistically analyzed with GraphPad Prism 5 statistical software (GraphPad Software, San Diego, CA) and expressed as mean \pm SD. Significance of the data obtained was assessed with Student's $t$-test (statistical significance was set at $P \leq 0.05)$.

\section{Results}

3.1. Morphological Study. Principal capillaroscopic abnormalities (megacapillaries, haemorrhages, ramifications, and avascular areas) resulted similar in Pso, PsA and RA patients $(P$-values $=$ N.S. $)$.

3.2. Morphometric Study. Capillaries of patients with Pso, PsA, and RA showed, respectively, a venous limb diameter of $17.6 \mu \mathrm{m} \pm 5.2,18 \mu \mathrm{m} \pm 5$ and $22 \mu \mathrm{m} \pm 4$ and an arterial limb diameter of $15.3 \mu \mathrm{m} \pm 4.3,15 \mu \mathrm{m} \pm 3$, and $16 \mu \mathrm{m} \pm 4$. Capillary loop diameter was $26.8 \mu \mathrm{m} \pm 6.3$ in Pso, $27 \mu \mathrm{m} \pm 7$ in PsA and equal to $30 \mu \mathrm{m} \pm 8$ in RA patients. The capillary loop amplitude was of $42.2 \mu \mathrm{m} \pm 10.3$ in Pso, $56 \mu \mathrm{m} \pm 12$ in RA, $40 \mu \mathrm{m} \pm 9$ in PsA. Linear density of capillaries was $13.2 \pm 2.1$ in Pso, $13 \pm 1$ in PsA, and $16 \pm 2$ in RA.

The number of twisted capillaries on $4 \mathrm{~mm}$ was of $10.5 \pm$ 2.1 in Pso, $12.2 \pm 3.2$ in PsA, and $7.1 \pm 1.2$ in RA patients. Comparative analysis of patients affected by Pso and PsA, with the control group showed significant statistical differences in three of considered parameters (amplitude of the capillary loop, linear density of capillaries, and number of twisted capillaries) with no differences between Pso and PsA. Capillaries of RA patients showed significant statistical differences if compared with the control group in venous limb 
TABLE 2: Patients demographic and clinical characteristics.

\begin{tabular}{lcccc}
\hline & Pso & PsA & RA & Controls \\
\hline Patients (number) & 30 & 30 & 30 & 30 \\
Gender (male/female) & $14 / 16$ & $11 / 19$ & $12 / 18$ & $15 / 15$ \\
Age & $48.1 \pm 12.3$ & $51.2 \pm 13.2$ & $53.1 \pm 13.6$ & $47.9 \pm 12.6$ \\
PASI score & $11.1 \pm 3.1$ & $9.6 \pm 2.8$ & 0 & 0 \\
DAS-28 ESR & 0 & $4.1 \pm 1.9$ & $4.9 \pm 2.2$ & 0 \\
CyA tp & $11 / 30$ & $3 / 30$ & $2 / 30$ & 0 \\
MTX tp & $6 / 30$ & $15 / 30$ & $19 / 30$ & 0 \\
Disease duration & $19.7 \pm 4.1$ & $11.6 \pm 3.3$ & $10.7 \pm 4$ & 0 \\
\hline
\end{tabular}

Data are expressed as mean \pm SD; PASI: psoriasis area severity index; DAS28-ESR: 28-joint disease activity score; CyA: cyclosporine A; MTX: methotrexate.

TABLE 3: Morphometric study results.

\begin{tabular}{|c|c|c|c|c|c|c|}
\hline & $\begin{array}{l}\text { Venous limb } \\
\text { diameter }\end{array}$ & $\begin{array}{l}\text { Arterial limb } \\
\text { diameter }\end{array}$ & Loop diameter & Loop amplitude & $\begin{array}{l}\text { Linear density } \\
\text { (Loops/2 mm) }\end{array}$ & $\begin{array}{c}\text { Tortuosity } \\
\text { (Twisted capil- } \\
\text { laries } / 4 \mathrm{~mm} \text { ) }\end{array}$ \\
\hline Pso & $17.6 \mu \mathrm{m} \pm 5.2$ & $15.3 \mu \mathrm{m} \pm 4.3$ & $26.8 \mu \mathrm{m} \pm 6.3$ & $42.2 \mu \mathrm{m} \pm 10.3$ & $13.2 \pm 2.1$ & $10.5 \pm 2.1$ \\
\hline PsA & $18 \mu \mathrm{m} \pm 5.4$ & $15.1 \mu \mathrm{m} \pm 3$ & $27.2 \mu \mathrm{m} \pm 7.1$ & $40.4 \mu \mathrm{m} \pm 9.1$ & $13.1 \pm 1$ & $12.2 \pm 3.2$ \\
\hline RA & $22 \mu \mathrm{m} \pm 4.1$ & $16.2 \mu \mathrm{m} \pm 4.1$ & $30 \mu \mathrm{m} \pm 8$ & $56 \mu \mathrm{m} \pm 12$ & $16 \pm 2$ & $7.1 \pm 1.2$ \\
\hline Controls & $17.5 \mu \mathrm{m} \pm 3.1$ & $14 \mu \mathrm{m} \pm 3.2$ & $26.4 \mu \mathrm{m} \pm 5$ & $46.1 \mu \mathrm{m} \pm 10$ & $16.4 \pm 2.6$ & $6.2 \pm 1.1$ \\
\hline Pso versus controls & N.S. & N.S. & N.S. & $P<0.001$ & $P<0.001$ & $P<0.001$ \\
\hline PsA versus controls & N.S. & N.S. & N.S. & $P<0.001$ & $P<0.001$ & $P<0.001$ \\
\hline RA versus controls & $P<0.001$ & $P<0.001$ & $P<0.001$ & $P<0.001$ & N.S & N.S. \\
\hline PsA versus RA & $P<0.001$ & $P<0.05$ & $P<0.001$ & $P<0.001$ & $P<0.001$ & $P<0.001$ \\
\hline Pso versus RA & $P<0.001$ & $P<0.05$ & $P<0.001$ & $P<0.05$ & $P<0.001$ & $P<0.001$ \\
\hline Pso versus PsA & N.S. & N.S. & N.S. & N.S. & N.S. & N.S. \\
\hline
\end{tabular}

Data are expressed as mean $\pm \mathrm{SD}$.

Statistical significance was assessed with Student's $t$-test; significance was set at $P<0.05$.

diameter, arterial limb diameter, capillary loop diameter, and amplitude of the capillary loop.

The study showed, furthermore, significant statistical differences between RA patients and psoriatic patients (with no differences between Pso and PsA) in all considered parameters.

Results are summarized in Table 3.

\section{Discussion}

Activation of vascular endothelium play a key role in initiation and progression of systemic inflammatory disease as psoriasis, psoriatic arthritis and rheumatoid arthritis. Deregulation of intercellular adhesion molecule-1 (ICAM-1) and vascular adhesion molecule-1 (VCAM-1) was detected in the skin and synovial membrane of patients with psoriasis and psoriatic arthritis [15].

Moreover, levels of VEGF (Vascular Endothelial Growth Factor) and sICAM (Soluble Intracellular Adhesion Molecules), considered to be biomarkers of vascular injury, have been found altered in course of chronic arthritis. There are controversial data about the role of VEGF in course of psoriasis and psoriatic arthritis. Experimental models of its inhibition seem to reduce inflammation; on the other hand the human use of Bevacizumab, a humanized monoclonal antibody that inhibits VEGF-A, did not show positive effects on psoriasis and there are case reports of reactivation of PSO and PsA in course of treatment.

In addition, VEGF and molecules membership are closely associated with the activity of key inflammatory molecules involved in psoriasis such as TNF and NFKB $[16,17]$.

Significant decrease of MMP-9 and MMP-2 levels in the sera was associated with clinical improvement and with the decrease of TNF- $\alpha$, VEGF, and E-selectin, angiogenic molecules already known to be implicated in clinical expression of psoriasis [18].

In the present study, morphometric analysis showed statistically significant differences between PsA, Pso, and RA.

In RA, measures of the venous limb diameter (efferent branch of the capillary loop), arterial limb diameter (afferent branch of the capillary loop), and loop diameter were significantly higher than in PsA, Pso, and the control group. The trend toward expansion, found in the capillaries of RA patients, is more evident at the efferent branch of the loop and may represent an early manifestation of vascular damage in the microcirculation [19].

In 1994, Hachulla et al., showed microvascular permeability alterations in RA, to confirming the existence of a microangiopathy [7]. 


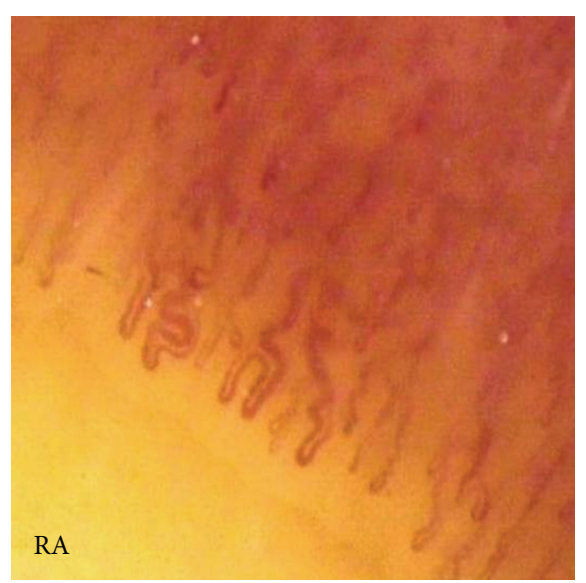

(a)

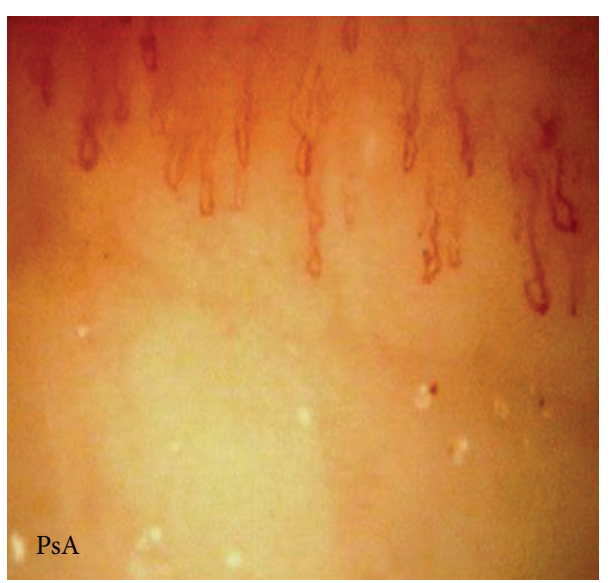

(b)

FIgURE 2: Capillaroscopic images of patients with RA and PsA. PsA patient has a higher number of twisted capillaries and a lower density if compared with RA patient.

In addition, Meyer et al. showed modifications of the normal blood flow velocity and microvascular dysfunction in RA [20].

Zaric et al., in a study conducted in patients with RA and PsA, reported capillaroscopic differences between the two diseases [8]. However, the differences concerned only the linear density of capillaries and measurements of the average diameter of the capillary segments of the loop were not carried out.

A study conducted on PsA patients, in agreement with our observations, showed a reduction in capillaries linear density [6].

It can be hypothesized that the density of the capillaries is reduced in patients suffering from psoriasis as a result of changes in the normal angiogenesis. On the other hand the loss of normal binding between the endothelial cell and matrix of the papillary dermis could explain the capillary branching and tortousity we detected in these patients.

The finding of a reduction of density in psoriatic patients is partially in contrast with those reported in studies conducted on the psoriatic plaque where a slight increase of capillary density was detected [21].

We believe that this observation is directly linked to the changes of the capillary in course of psoriasis (tendency to terminal tortuosity and branching).

During microscopic observation of psoriasis plaque, indeed, capillaries are shown frontally so it is possible that ramifications and tortuosity (typically associated with psoriasis) are incorrectly interpreted as individual capillaries rather than ramifications of the same capillary. This artefact is absent in the periungual study because the longitudinal view allows to follow the entire course of capillary within the dermal papilla.

In our opinion, the increase in the perfusion of the plaque is connected to a vasodilation and an increase of the flow velocity rather than to an increased density of capillaries.

In conclusion, in RA patients we found capillaries with larger diameters if compared with those of psoriatic patients and the control group. We also observed a reduction in the linear density of the capillaries and an increased tortuosity in psoriatic patients than the other two groups. These morphologic characteristics may reflect pathogenetic differences between the two diseases providing an additional approach to establish a correct differential diagnosis (Figure 2).

In our opinion capillaroscopy can be considered a valid technique in inflammatory joint diseases to analyze microvascular circulation.

\section{References}

[1] E. Christophers, "Psoriasis-epidemiology and clinical spectrum," Clinical and Experimental Dermatology, vol. 26, no. 4, pp. 314-320, 2001.

[2] D. Kane, L. Stafford, B. Bresniham, and O. FitzGerard, "A prospective, clinical and radiological study of early psoriatic arthritis: an early synovitis clinic experience," Rheumatology, vol. 42, no. 12, pp. 1460-1468, 2003.

[3] G. Girolomoni and P. Gisondi, "Psoriasis and systemic inflammation: underdiagnosed enthesopathy," Journal of the European Academy of Dermatology and Venereology, vol. 23, supplement 1, pp. 3-8, 2009.

[4] P. J. Mease, C. E. Antoni, D. D. Gladman, and W. J. Taylor, "Psoriatic arthritis assessment tools in clinical trials," Annals of the Rheumatic Diseases, vol. 64, supplement 2, pp. ii49-ii54, 2005.

[5] C. Alessandri, R. Priori, M. Modesti, R. Mancini, and G, Valesini, "The role of anti-cyclic cytrullinate antibodies testing in rheumatoid arthritis," Clinical Reviews in Allergy and Immunology, vol. 34, no. 1, pp. 45-49, 2008.

[6] M. Bhushan, T. Moore, A. L. Herrick, and C. E. M. Griffiths, "Nailfold video capillaroscopy in psoriasis," The British Journal of Dermatology, vol. 142, no. 6, pp. 1171-1176, 2000.

[7] E. Hachulla, M. Perez-Cousin, R. M. Flipo et al., "Increased capillary permeability in systemic rheumatoid vasculitis: detection by dynamic fluorescence nailfold videomicroscopy," Journal of Rheumatology, vol. 21, no. 7, pp. 1197-1202, 1994. 
[8] D. Zaric, A. M. Worm, D. Stahl, and O. J. Clemmensen, "Capillary microscopy of the nailfold in psoriatic and rheumatoid arthritis," Scandinavian Journal of Rheumatology, vol. 10, no. 3, pp. 249-252, 1981.

[9] C. Cervini and W. Grassi, "Periungual capillary microscopy in patients with rheumatoid arthritis and psoriatic arthritis," Zeitschrift fur Rheumatologie, vol. 43, no. 6, pp. 311-313, 1984.

[10] W. Grassi, P. Core, G. Carlino, and C. Cervini, "Nailfold capillary permeability in psoriatic arthritis," Scandinavian Journal of Rheumatology, vol. 21, no. 5, pp. 226-230, 1992.

[11] M. Cutolo, C. Pizzorni, and A. Sulli, "Capillaroscopy," Best Practice and Research: Clinical Rheumatology, vol. 19, no. 3, pp. 437-452, 2005.

[12] S. N. Lambova and U. Müller-Ladner, "Capillaroscopic pattern in inflammatory arthritis," Microvascular Research, vol. 83, no. 3, pp. 318-322, 2012.

[13] W. Taylor, D. Gladman, P. Helliwell, A. Marchesoni, P. Mease, and H. Mielants, "Classification criteria for psoriatic arthritis: development of new criteria from a large international study," Arthritis and Rheumatism, vol. 54, no. 8, pp. 2665-2673, 2006.

[14] D. J. McShane, J. F. Fries, N. S. Cooper et al., "The American rheumatism association 1987 revised criteria for the classification of rheumatoid arthritis," Arthritis and Rheumatism, vol. 31, no. 3, pp. 315-324, 1988.

[15] D. Veale, S. Rogers, and O. Fitzgerald, "Immunolocalization of adhesion molecules in psoriatic arthritis, psoriatic and normal skin," The British Journal of Dermatology, vol. 132, no. 1, pp. 3238, 1995.

[16] H. B. Schonthaler, R. Huggenberger, S. K. Wculek, M. Detmar, and E. F. Wagner, "Systemic anti-VEGF treatment strongly reduces skin inflammation in a mouse model of psoriasis," Proceedings of the National Academy of Sciences of the United States of America, vol. 106, no. 50, pp. 21264-21269, 2009.

[17] D. Graceffa, E. Maiani, A. Pace et al., "Psoriatic arthritis during treatment with bevacizumab for anaplastic oligodendroglioma," Case Reports in Rheumatology, vol. 2012, Article ID 208606, 3 pages, 2012.

[18] P. Cordiali-Fei, E. Trento, G. D’Agosto et al., "Effective therapy with anti-TNF- $\alpha$ in patients with psoriatic arthritis is associated with decreased levels of metalloproteinases and angiogenic cytokines in the sera and skin lesions," Annals of the New York Academy of Sciences, vol. 1110, pp. 578-589, 2007.

[19] A. Kuryliszyn-Moskal, "Cytokines and soluble CD4 and CD8 molecules in rheumatoid arthritis: relationship to systematic vasculitis and microvascular capillaroscopic abnormalities," Clinical Rheumatology, vol. 17, no. 6, pp. 489-495, 1998.

[20] M. F. Meyer, O. Schmidt, B. Hellmich, H. Schatz, H. H. Klein, and J. Braun, "Microvascular dysfunction in rheumatoid arthritis assessed by laser Doppler anemometry: relationship to soluble adhesion molecules and extraarticular manifestations," Rheumatology International, vol. 28, no. 2, pp. 145-152, 2007.

[21] P. Rosina, A. Giovannini, P. Gisondi, and G. Girolomoni, "Microcirculatory modifications of psoriatic lesions during topical therapy," Skin Research and Technology, vol. 15, no. 2, pp. 135-138, 2009. 


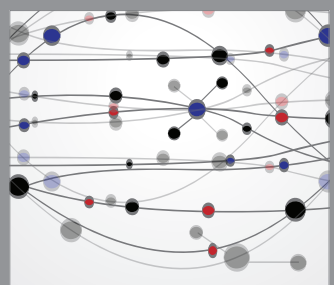

The Scientific World Journal
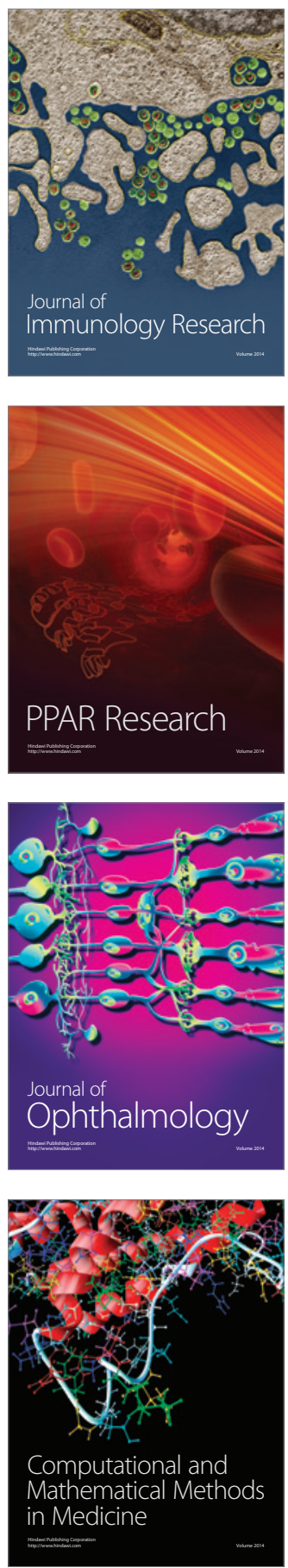

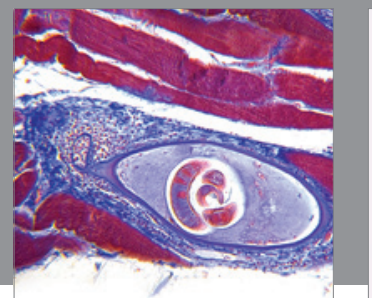

Gastroenterology

Research and Practice
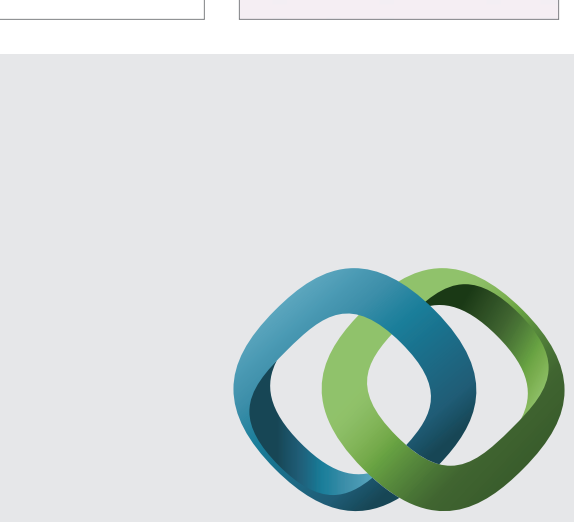

\section{Hindawi}

Submit your manuscripts at

http://www.hindawi.com
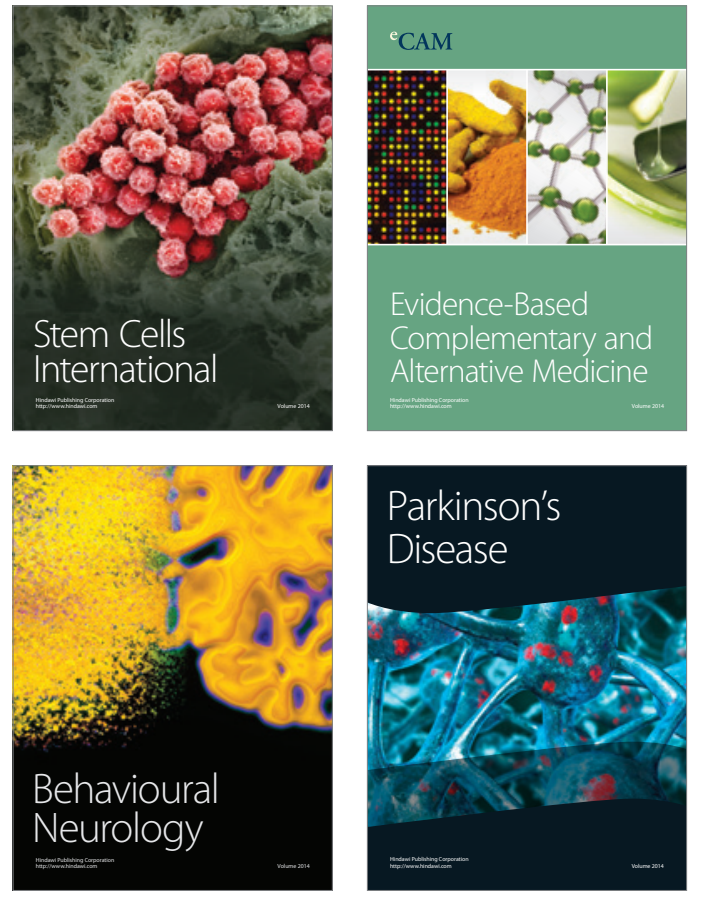
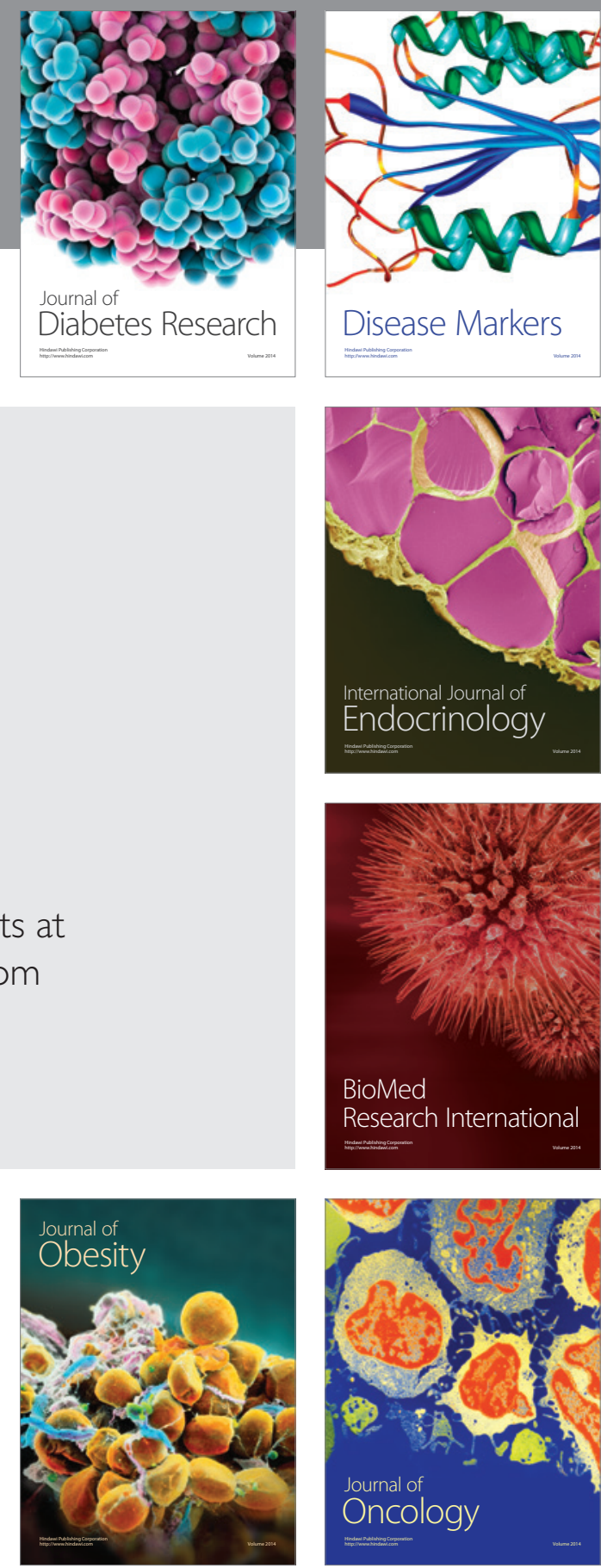

Disease Markers
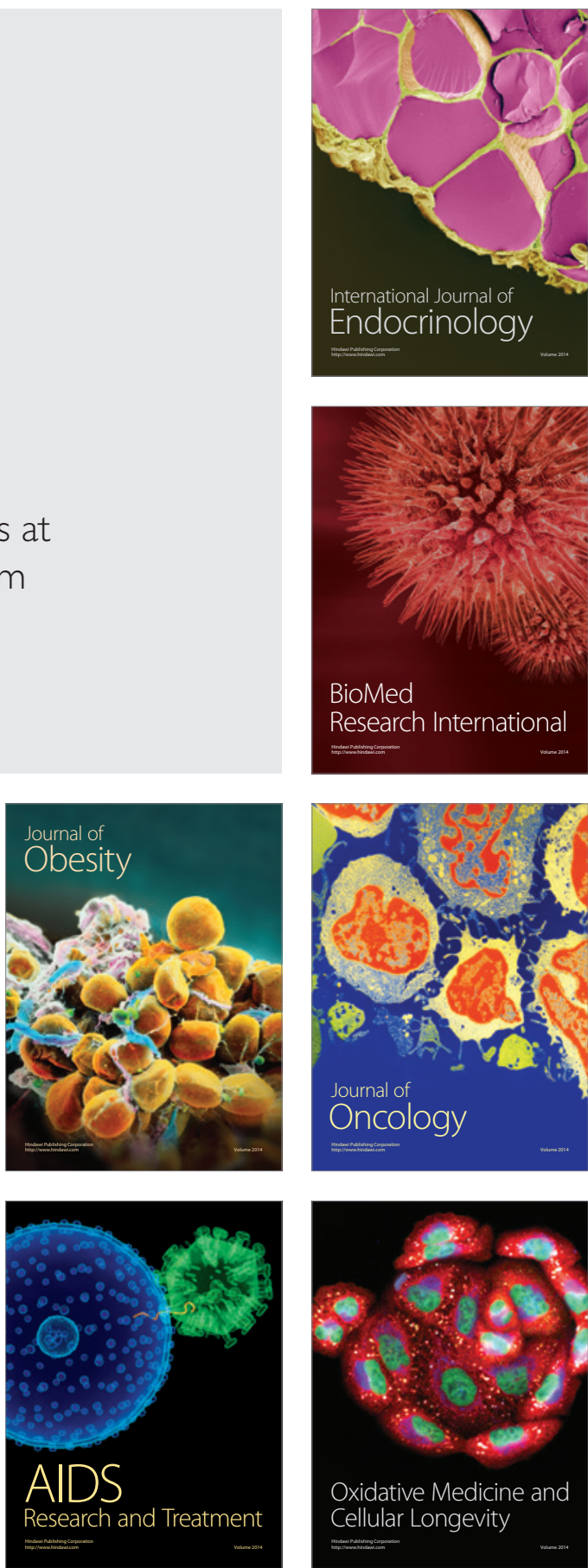